\title{
Temporal discounting and preference reversals in choice between delayed outcomes
}

\author{
LEONARD GREEN, NATHANAEL FRISTOE, and JOEL MYERSON \\ Washington University, St. Louis, Missouri
}

\begin{abstract}
Subjects chose between pairs of hypothetical amounts of money available after different delays. When smaller, more immediate amounts were selected over larger, more delayed amounts, the addition of a constant delay to both outcomes resulted in reversals of preference, contrary to the standard discounted utility model of economics. The delays at which preference reversed were determined for three pairs of amounts ( $\$ 20$ vs. $\$ 50, \$ 100$ vs. $\$ 250$, and $\$ 500$ vs. $\$ 1,250$ ). The relation between the delay to the larger amount and the delay to the smaller amount at preference reversal was well fit by both linear and quadratic functions. Intercepts increased with amount, strongly suggesting that rate of discounting decreases with amount. The presence of significant negative curvature in the data from the majority of individual subjects poses problems for exponential and hyperbolic models of temporal discounting in self-control, both of which predict a linear relation between the delays to the larger and smaller amounts.
\end{abstract}

Given a choice between a smaller reward available after a brief delay and a larger reward available only after a long delay, an individual might choose the smaller reward. For example, $\$ 100$ available tomorrow might be preferred to $\$ 200$ available in 2 months. However, if the delays to both rewards were increased equally, preference might reverse, and the individual might choose the larger, more delayed reward. Continuing with our example, $\$ 200$ available in 8 months (an increase of 6 months) might be preferred to $\$ 100$ available in 6 months and a day (also a 6-month increase).

Many everyday decisions involve choices between delayed rewards: getting a lower paying job right out of high school versus going to college in order to obtain a higher paying job after graduation; choosing between two certificates of deposit, one that matures sooner versus one that matures later but pays a higher rate of interest. In addition, choices between delayed rewards lie at the heart of contemporary accounts of impulsivity and self-control (e.g., Ainslie, 1975, 1992; Rachlin, 1974, in press). The phenomenon of preference reversal provides an important part of the empirical foundation for these accounts, and there is a growing body of literature in the fields of economics and psychology on this topic (for examples, see the edited volume by Loewenstein \& Elster, 1992).

Reversals in preference between delayed rewards have typically been explained in terms of the discounting of the subjective value of rewards over time. Figure 1 illustrates how changes in the value of rewards over time can account for preference reversals. The bars represent two

Nathanael Fristoe is now at the Georgia Institute of Technology, Atlanta. Requests for reprints should be directed to L. Green, Department of Psychology, Washington University, Campus Box 1125, St. Louis, MO 63130. rewards, one large and one small, and the curves show how their (subjective) values change as a function of the delays until they are received. As can be seen, an individual faced with a choice between two future rewards, the smaller of which can be obtained sooner, might reach different decisions depending upon when the choice is made. If the choice is made at time $T_{1}$, the smaller reward will be chosen. If the delays to both rewards are increased equally so that the choice is made at time $T_{2}$, preference reverses, and the larger, more delayed reward will be chosen.

The preference-reversal phenomenon has provided the basis for inferences regarding the form of the discount function - that is, the function relating the subjective value of a reward to the delay until its receipt (e.g., the curves in Figure 1). If the shape of the discount function is hyperbolic such that

$$
V=A /(1+k D),
$$

where $V$ is the subjective value and $A$ is the amount of the delayed reward, $D$ is the delay to its receipt, and $k$ is a parameter governing the rate at which value is discounted, then preference reversals are predicted (Ainslie, 1975, 1992). In contrast, if the shape of the discount function is exponential such that

$$
V=A * \exp (-k D),
$$

then the ratio of the values of the two rewards remains constant as their delays are increased equally, and no change in preference is predicted. Given that preferences do reverse (e.g., Green, Fisher, Perlow, \& Sherman, 1981; Navarick, 1982), an account based on exponential discounting has been rejected (Ainslie, 1975. 1992; Mazur, 1987; Rodriguez \& Logue, 1988).

However, this argument against exponential discounting is based on the assumption that the value of the dis- 


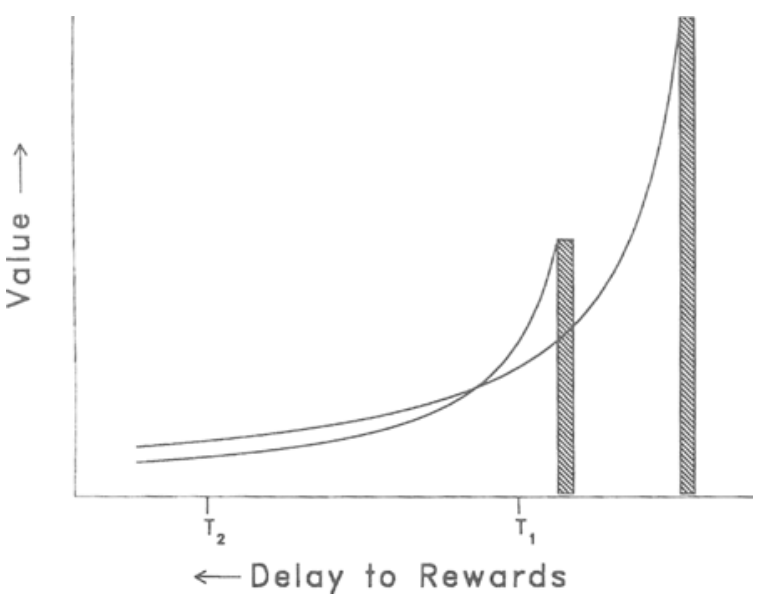

Figure 1. Subjective value of two hyperbolically discounted rewards that differ in both amount and delay. The shorter bar represents the smaller, more immediate reward, and the taller bar represents the larger, more delayed reward. The curves represent value as a function of the time until receipt.

count rate parameter $(k)$ is the same for small and large rewards. Given this assumption, the exponential model predicts that preferences will not reverse, but the model does predict preference reversals if the value of $k$ is inversely related to amount of reward (Green \& Myerson, 1993). Indeed, recent studies have shown an inverse relation between $k$ and amount of reward such that larger amounts are discounted less steeply than smaller amounts (Benzion, Rapoport, \& Yagil, 1989; Green, Fry, \& Myerson, 1994; Raineri \& Rachlin, 1993; Thaler, 1981).

In addition to the argument based on preference reversals, there is a second line of argument against exponential discount functions, and for hyperbolic discount functions. This second argument focuses on the relation between the delays to the smaller and larger rewards at the points at which the two rewards are judged to be equivalent in value. Mazur (1987) has argued that hyperbolic discounting predicts that this relation will be linear with a slope greater than 1.0 , whereas exponential discounting predicts that the slope will be equal to 1.0. A linear relation between the delays to the smaller and larger rewards at points of equivalent value has been demonstrated in both pigeons and humans (Mazur, 1987; Rodriguez \& Logue, 1988), and the slopes were greater than 1.0 .

However, Mazur's argument, like that leading to the rejection of exponential discounting because of preference reversals, is based on the assumption that the value of the discount rate parameter is the same for small and large rewards. If the value of $k$ is inversely related to amount of reward, then both exponential discounting and hyperbolic discounting models predict a linear relation with a slope greater than 1.0. Moreover, both models predict that if the absolute amounts increase but their ratio is held constant, the intercept of the relation between delays to larger and smaller rewards will increase.
In contrast, if $k$ is independent of amount, the intercept will remain constant as the absolute amounts increase as long as their ratio is constant. (The derivation of these predictions is given in the Appendix.)

Thus, the relation between the delays to larger and smaller rewards at the points where the rewards are equal in subjective value does not discriminate between exponential and hyperbolic models when the $k$ parameter varies inversely with amount. Nevertheless, this relation does provide a test of the standard discounted utility model (Samuelson, 1937), which has provided the basis for much theoretical work by economists on choice between delayed rewards (Loewenstein, 1992). The discounted utility model assumes exponential discounting at a rate independent of amount and therefore predicts a slope of 1.0 .

Although the results of previous research are inconsistent with the slope prediction of the exponential model favored by economists, the range of delays examined in previous studies was very restricted (under $1 \mathrm{~min}$ ). Such delays are considerably shorter than those discussed in economic theory, which considers delays on the order of years (e.g., Friedman, 1957; Mohabbat \& Simos, 1977; Olson \& Bailey, 1981). In addition, in the one pertinent human study (Rodriguez \& Logue, 1988), rewards were opportunities to earn points worth less than a penny each, substantially less than the amounts considered by economists as well as those encountered in many everyday decisions.

In the present study, subjects made choices between hypothetical monetary rewards available after different delays. The ratio of the larger to the smaller amount was held constant $(\$ 20$ to $\$ 50 ; \$ 100$ to $\$ 250 ; \$ 500$ to $\$ 1,250$ ), and the delays were varied over an extended range (from no delay to a delay of 20 years). The points at which preference for the smaller, more immediate reward changed to preference for the larger, more delayed reward were determined. The experiment addressed two questions: First, does preference reverse as equal amounts of time are added to the delays to smaller and larger rewards when the amounts and delays under consideration are within the same range as those often encountered in everyday decision making? Second, is the relation between the delay to the larger reward and the delay to the smaller reward at the points of preference reversal consistent with the predictions of exponential and hyperbolic discounting models?

\section{METHOD}

\section{Subjects}

Twenty-four undergraduates, 9 males and 15 females, participated. They were informed that the purpose of the study was to examine preferences between hypothetical monetary rewards and were paid $\$ 5$ for participating.

\section{Procedure}

Participants sat at a table and made a series of choices between two hypothetical monetary rewards obtainable at different delays. Three pairs of rewards were used: $\$ 20$ versus $\$ 50, \$ 100$ versus $\$ 250$, and $\$ 500$ versus $\$ 1,250$. The dollar amounts were printed on $5 \times 7$ in 
cards. The card showing the small amount was presented to the subject's left, and the card showing the large amount to the subject's right. The delay durations at which these amounts could be received were printed on $4 \times 6$ in. cards presented below the cards indicating the amounts. The smaller reward was available either immediately or after a delay of 1 week, 2 weeks, 1 month, 3 months, 6 months, 1 year, 2 years, 3 years, 5 years, 7 years, 10 years, 12 years, 15 years, 17 years, or 20 years. The larger reward was available at the sum of the delay to the smaller reward plus one of the following interreward delays: 1 week, 1 month, 3 months, 6 months, 1 year, 3 years, 5 years, 7 years, 10 years, 15 years, or 20 years.

Participants received the following instructions:

The purpose of this study is to examine your preferences for different hypothetical dollar amounts. You will be asked to make a series of decisions between monetary altematives. In making your decision, it is important to read carefully the cards which appear in front of the dollar amounts because they specify when the money will be made available. Please indicate your choice by pointing to the dollar-amount card you prefer under the conditions stated. Remember, there are no right or wrong answers in this study. After you have made your decision, the experimenter will turn the cards over for the next trial. You will be given four practice trials before you begin the real trials. Once the trials begin, the experimenter will no longer be able to answer any questions, so it is important that you fully understand the procedure at this time.

A hypothetical example of the procedure is outlined in Figure 2. The experimenter initially determined the briefest interreward delay at which a subject preferred the smaller reward when it was available immediately (Steps 1-3 in Figure 2). Then the delays to both rewards were increased equally until the subject's preference reversed and the larger reward was preferred for two consecutive choices (Steps 4-7). Next, the subject was given a choice between the smaller amount received immediately and the larger amount available at the next longer interreward delay (Step 8), and the delays to both rewards were again increased equally until preference reversed once more (Steps 9, 10, ...). This procedure was repeated until a subject had been tested at all eleven interreward delays for each of the three pairs of amounts. Participation took less than an hour. The order in which the three amount pairs were presented followed a Latin square design.

\section{RESULTS}

In order to examine the effects of equally incrementing the delays to the two rewards, the percentage of subjects choosing the larger, more delayed reward was calculated at each value of the delay to the smaller reward. Figure 3 shows these percentages separately for each interreward delay. The top, middle, and bottom panels present the data for the small ( $\$ 20$ vs. $\$ 50)$, medium $(\$ 100$ vs. $\$ 250)$, and large ( $\$ 500$ vs. $\$ 1,250)$ reward pairs. Although each panel shows the data from all 11 interreward delays, there was considerable overlap in the results at the briefer interreward delays ( 3 years or less), and therefore only selected delays are labeled.

As the delay to the smaller reward was lengthened, the percentage of subjects choosing the larger, more delayed reward increased. This effect was observed for each of the three reward pairs and at every interreward delay. At the briefer interreward delays (e.g., 1 week to 3 years), the percentage choosing the larger reward increased sharply as choice delay increased, leveling off at a high value. At larger interreward delays (e.g., 5 or more years), the percentage increased more slowly and either did not reach an asymptote or leveled off at a lower value.

In order to examine the relation between the delays to the two amounts at the points of preference reversal, the delay to the larger amount was plotted as a function of
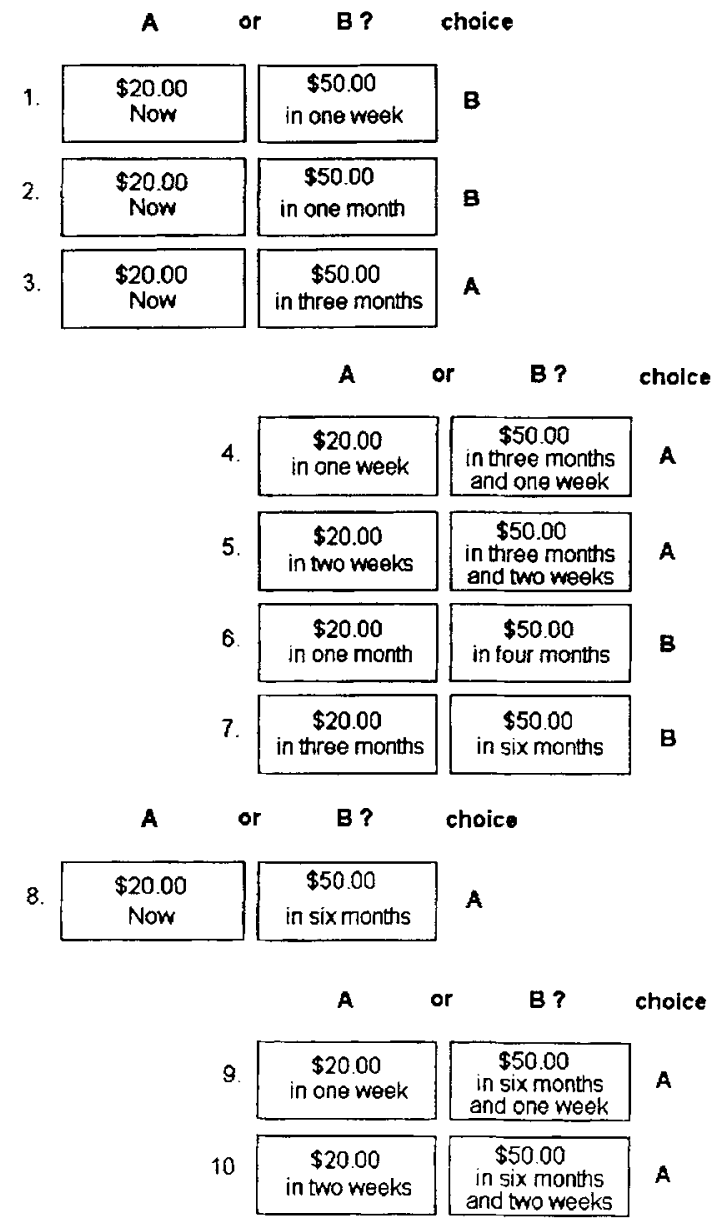

etc

Figure 2. Example of the procedure used in the present study.

the delay to the smaller amount. At each interreward delay, we determined the median delay to the smaller amount at the point of preference reversal. The corresponding delay to the larger amount was equal to the interreward delay plus the delay to the smaller amount. The top panel of Figure 4 presents the delays to the two amounts at the point of preference reversal for the three amount pairs.

At the point of preference reversal, the smaller, more immediate amount is assumed to be subjectively equivalent in value to the larger, more delayed amount. Thus, Figure 4 shows how long the delay to the larger amount must be in order for this amount to be judged equivalent in value to a smaller amount that is available sooner. Polynomial regression was used to test whether the relations between the delays were linear, as predicted by both exponential and hyperbolic discounting models. Although linear regression provided very good fits to the data for all three amount pairs, in each case polynomial regression revealed a significant quadratic component (all $p \mathrm{~s}<.02$ ), indicating the presence of nonlinearity. Second-order polynomials provided extremely 


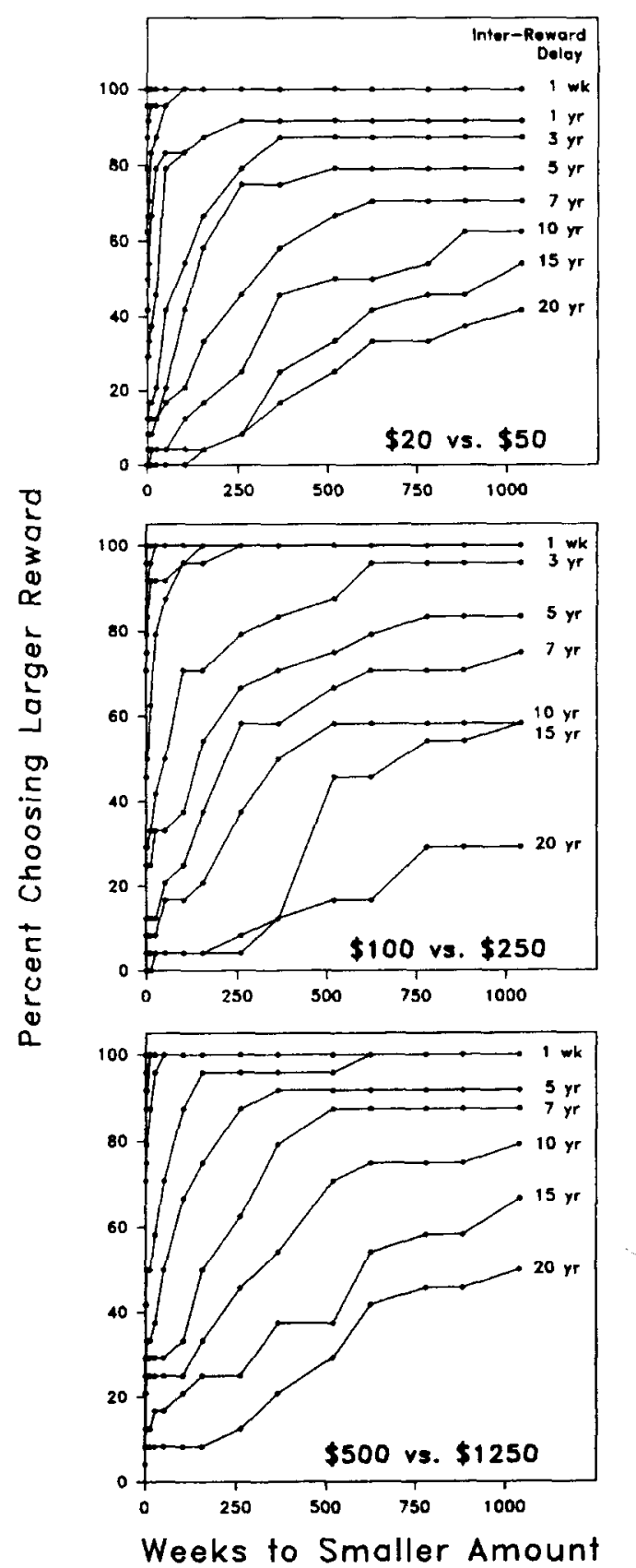

Figure 3. Percentage of subjects choosing the larger, more delayed reward as a function of delay until the smaller reward. Individual lines connect the data for each interreward delay. Results obtained with each of the three pairs of amounts are shown in separate panels.

accurate descriptions of the data (all $R^{2} \mathrm{~s}>.99$ ). The parameters of the best-fitting polynomials and the proportions of explained variance for the small, medium, and large amount pairs, as well as for the mean of the three pairs, are given in Table 1 . The fit of a negatively accelerated polynomial to the mean data is shown in the bottom panel of Figure 4. It can be seen that the negative curvature is not simply due to the large range of delays examined; curvature is equally apparent when only delays of up to 7 years are considered.

Consistent with the parallel appearance of the relations shown in the top panel of Figure 4, there were no systematic differences between either the first- or the second-order regression coefficients for the three pairs (see Table 1). However, the intercepts did increase systematically as a function of absolute amount. A secondorder polynomial model that included a separate zeroorder coefficient (i.e., a separate intercept term) for each of the three amount pairs, but common first- and secondorder coefficients, accounted for more than $99 \%$ of the variance and provided a significantly better description of the data than did a simple polynomial with a single intercept for all three pairs $[F(2,43)=54.98, p<.001]$.

The data from each of the 24 subjects, averaged across the three pairs of amounts, were also analyzed by

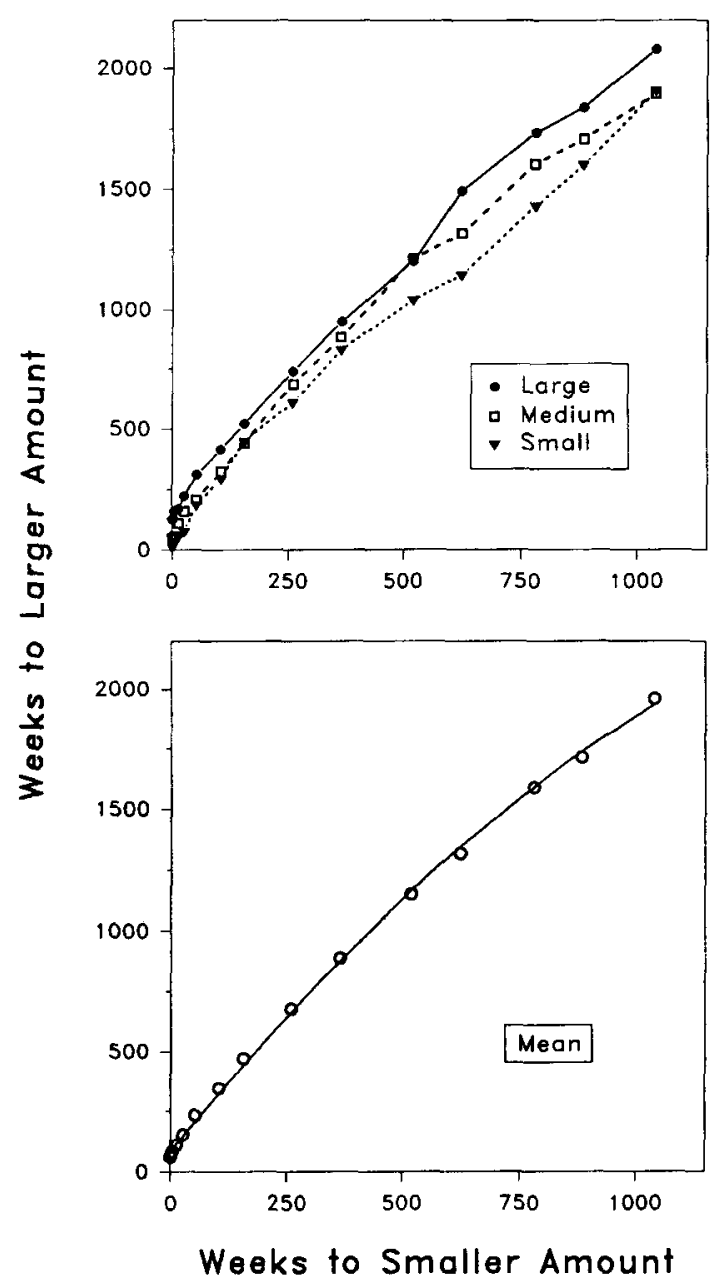

Figure 4. Delay to the larger reward as a function of delay to the smaller reward at the point where both rewards are subjectively equivalent in value. Top: Median equivalence points are shown separately for each of the three pairs of amounts. Bottom: Means averaged across the three pairs of amounts are shown, as well as the bestfitting polynomial function. 
Table 1

Polynomial Regressions for the Small, Medium, and Large Amount Pairs

\begin{tabular}{llc}
\hline \multicolumn{1}{c}{ Amounts } & \multicolumn{1}{c}{ Equation } & $R^{2}$ \\
\hline$\$ 20$ vs. $\$ 50$ & $y=44.1+2.14 x-.0004 x^{2}$ & .995 \\
$\$ 100$ vs. $\$ 250$ & $y=61.9+2.57 x-.0008 x^{2}$ & .999 \\
$\$ 500$ vs. $\$ 1,250$ & $y=146.0+2.44 x-.0006 x^{2}$ & .999 \\
Mean & $y=84.0+2.38 x-.0006 x^{2}$ & .999 \\
\hline
\end{tabular}

using polynomial regression. In order to determine whether there was significant negative acceleration at the individual level, a $t$ test based on the individual coefficients for the second-order (quadratic) component was conducted. The test revealed that, consistent with the hypothesis of negative acceleration in the individual functions, these coefficients were significantly less than zero $[t(22)=-3.48, p<.01]$. Figure 5 presents the data and best-fitting polynomials, plotted as in Figure 4 , for 10 of the subjects. The subjects represented are those who showed a preference reversal at each of the delay values studied, thereby providing the most data points with which to assess the form of the function. In 7 of these 10 individuals, the relation between the delays to the larger and smaller amounts shows statistically significant negative acceleration (all seven $t s<-2.3$, ps $<$ .05 ). There was also significant negative curvature in 8 of the individual cases that are not shown. Thus, the nonlinear relation in the group data, shown in the bottom panel of Figure 4, is representative of the results at the individual level and not an artifact of averaging.

\section{DISCUSSION}

The present results are consistent with a choice process similar to that represented in Figure 1. That is, the results support a process in which the value of rewards is discounted over time and in which the discount functions for larger and smaller rewards cross as the delays to both rewards are incremented equally. For a given interreward delay at which preference was initially for the smaller amount, incrementing the delays to both rewards equally resulted in a switch in preference to the larger, more delayed reward. In addition, greater increments were required to produce preference reversals at longer interreward delays, also consistent with the process in Figure 1. The present findings demonstrate that the addition of equal amounts of time before the receipt of the smaller and larger rewards results in preference reversals. Importantly, our results show that such preference reversals occur under conditions in which the values of the delays and amounts are within the range of those involved in much everyday decision making.

Although these findings are predicted by recent psychological analyses of choice between delayed reinforcers (Ainslie, 1992; Green et al., 1994; Logue, 1988; Mazur, 1987; Rachlin, Raineri, \& Cross, 1991), they are inconsistent with the amount-insensitive exponential discount functions assumed by the standard discounted utility theory of economics (Samuelson, 1937; Loewenstein, 1992). Such discount functions do not cross as the delays to rewards increase. Hence, economic theory predicts that preferences will not reverse in situations like those studied here. This problem with economic theory has been pointed out previously, beginning with Bohm-Bawerk (1923), and it is highlighted in a classic article by Strotz (1956). However, economists have not yet reached a general consensus on the resolution of this difficulty (Loewenstein \& Elster, 1992).

The present findings are qualitatively consistent with the predictions of psychological analyses. However, certain quantitative limitations are evident. The hyperbolic discount functions favored by psychologists (as well as the exponential discount functions assumed by economists) predict a linear relation between the delays to the larger and smaller rewards at the point of preference reversal (see the Appendix for derivation). This linearity provides the starting point for a new discount function having the form of a generalized hyperbola that has been proposed by Loewenstein and Prelec (1992). Although linear functions provide very good fits to the present data, the relation between the delays to the larger and smaller rewards is clearly nonlinear, both at the group level and in the majority of individual cases.

On the other hand, even though they fail to predict the precise form of the relation between the two delays, hyperbolic and exponential discount models in which discount rates are inversely related to amount of reward both capture important aspects of the data. First, both forms of discount function correctly predict that preference will reverse when the delays to both the larger and smaller rewards are increased (Green

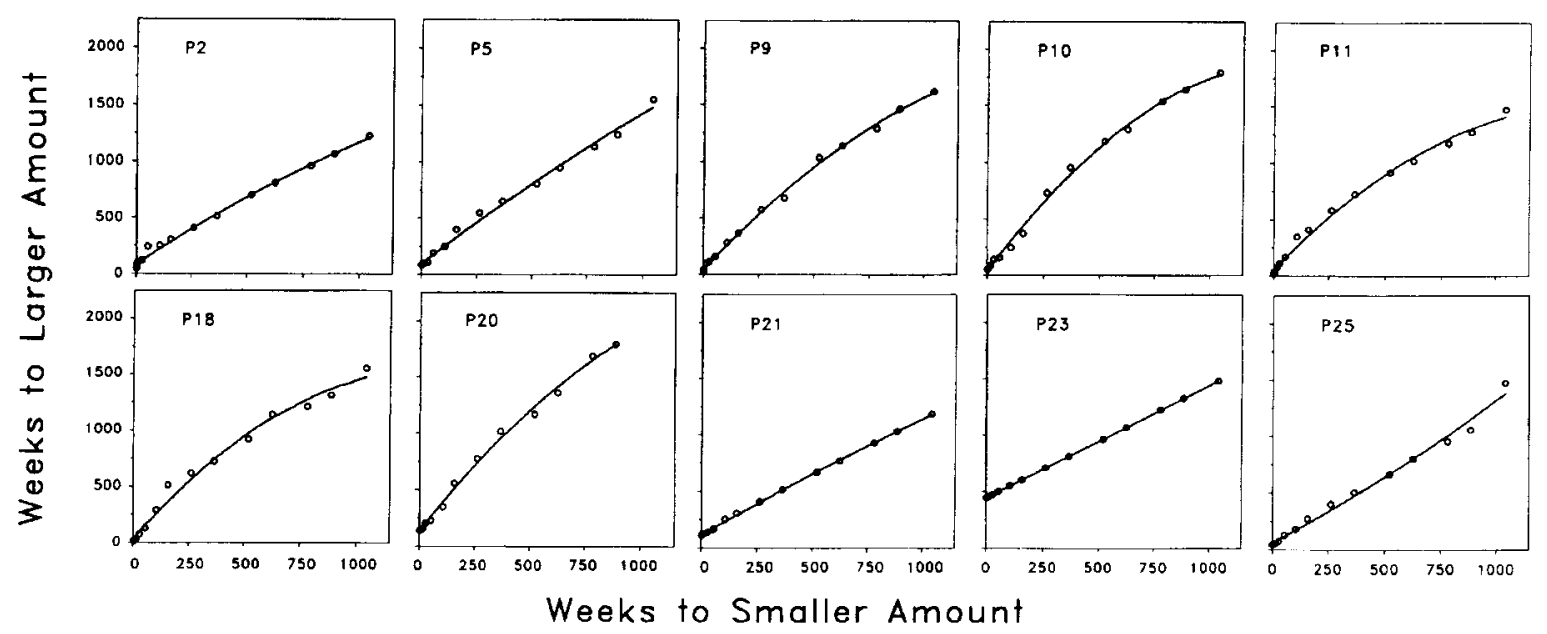

Figure 5. Delay to the larger reward as a function of delay to the smaller reward at the point where both rewards are subjectively equivalent in value. Data from 10 individuals are shown in separate panels. For each subject, means averaged across the three pairs of amounts are shown, as well as the best-fitting polynomial function. 
\& Myerson, 1993). Second, both correctly predict that, with the ratio of amounts held constant, the function relating the two delays will intercept the $y$-axis at higher values as the absolute amounts of the rewards increase. If $k$ were independent of amount, both models would predict that a constant ratio would lead to a constant intercept. Thus, the increase in intercept with absolute amount observed in the present study provides converging evidence of the fact that discount rate is dependent on amount, as observed in previous studies using quite different procedures and analytical techniques (Benzion et al., 1989; Green et al., 1994; Raineri \& Rachlin, 1993; Thaler, 1981).1

Given that discount rate is inversely related to amount, both hyperbolic and exponential models also correctly predict slopes greater than 1.0. That is, both predict that increases in the delay to the smaller reward will require even greater increases in the delay to the larger reward in order to maintain equivalence in the values of the two rewards, and this prediction was confirmed. Thus, both models capture important aspects of the data, once amount-dependent discounting rate is assumed. However, their failure to predict the negative acceleration that characterizes the relation between the two delays poses a potentially serious problem, one that must be addressed in future empirical and theoretical studies.

\section{REFERENCES}

AINSLIE, G. (1975). Specious reward: A behavioral theory of impulsiveness and impulse control. Psychological Bulletin, 82, 463-496.

AINSLIE, G. (1992). Picoeconomics: The strategic interaction of successive motivational states within the person. Cambridge: Cambridge University Press.

BENZION, U., RAPOPORT, A., \& YAGIL, J. (1989). Discounting rates inferred from decisions: An experimental study. Management Science, 35, 270-284.

BOHM-BAWERK, E. v. (1923). The positive theory of capital (W. Smart, Trans.). New York: G. E. Stechert. (Original work published 1888)

Friedman, M. (1957). A theory of the consumption function. Princeton, NJ: Princeton University Press.

Green, L., Fisher, E. B., Jr., Perlow, S., \& Sherman, L. (1981). Preference reversal and self-control: Choice as a function of reward amount and delay. Behaviour Analysis Letters, 1, 43-51.

Green, L., Fry, A., \& Myerson, J. (1994). Discounting of delayed rewards: A life span comparison. Psychological Science, 5, 33-36.

Green, L., \& Myerson, J. (1993). Alternative frameworks for the analysis of self control. Behavior \& Philosophy, 21, 37-47.

LOEWENSTEIN, G. (1992). The fall and rise of psychological explanations in the economics of intertemporal choice. In G. Loewenstein \& J. Elster (Eds.), Choice over time (pp. 3-34). New York: Russell Sage.

Loewenstein, G., \& Elster, J. (EDS.) (1992). Choice over time. New York: Russell Sage.

Loewenstein, G., \& Prelec, D. (1992). Anomalies in intertemporal choice: Evidence and an interpretation. Quarterly Journal of Economics, 107, 573-597.

LOGUE, A. W. (1988). Research on self-control: An integrating framework. Behavioral \& Brain Sciences, 11, 665-679.

MAZUR, J. E. (1987). An adjusting procedure for studying delayed reinforcement. In M. L. Commons, J. E. Mazur, J. A. Nevin, \& H. Rachlin (Eds.), Quantitative analyses of behavior: Vol. 5. The effect of delay and of intervening events on reinforcement value (pp. 55-73). Hillsdale, NJ: Erlbaum.

MohabBat, K. A., \& Simos, E. O. (1977). Consumer horizon: Further evidence. Journal of Political Economy, 85, 851-858.

NAVARICK, D. J. (1982). Negative reinforcement and choice in humans. Learning \& Motivation, 13, 361-377.

Olson, M., \& BaIley, M. J. (1981). Positive time preference. Journal of Political Economy, 89, 1-25.

RACHLIN, H. (1974). Self-control. Behaviorism, 2, 94-107.

RaChLiN, H. (in press). Self-control: Beyond commitment. Behavioral \& Brain Sciences.

RACHLIN, H., RAINERI, A., \& Cross, D. (1991). Subjective probability and delay. Journal of the Experimental Analysis of Behavior, 55, 233-244.
Raineri, A., \& RaChlin, H. (1993). The effect of temporal constraints on the value of money and other commodities. Journal of Behavioral Decision Making, 6, 77-94.

Rodriguez, M. L., \& Logue, A. W. (1988). Adjusting delay to reinforcement: Comparing choice in pigeons and humans. Journal of $E x-$ perimental Psychology: Animal Behavior Processes, 14, 105-117.

SAMUELSON, P. A. (1937). A note on measurement of utility. Review of Economic Studies, 4, 155-161.

STROTZ, R. H. (1956). Myopia and inconsistency in dynamic utility maximization. Review of Economic Studies, 23, 165-180.

Thaler, R. (1981). Some empirical evidence on dynamic inconsistency. Economic Letters, 8, 201-207.

\section{NOTE}

1. Indeed, intercept values may be used to estimate $k$ for the larger amount of a pair. Assuming hyperbolic discounting, the $k$ values for the present data were $.022, .014$, and .008 for the $\$ 50, \$ 250$, and $\$ 1,250$ rewards, respectively. Assuming exponential discounting, the $k$ values were $.013, .008$, and .005 , respectively.

\section{APPENDIX}

Assume that the subjective value, $V$, of a reward decreases hyperbolically with the delay until its receipt (Equation 1 ). If two rewards are equal in subjective value (i.e., $V_{1}=V_{2}$ ), then

$$
A_{1} /\left(1+k_{1} * D_{1}\right)=A_{2} /\left(1+k_{2} * D_{2}\right),
$$

where $A$ represents amount, $D$ represents delay, and $\mathrm{k}$ is the parameter that determines how steeply the value of a reward is discounted. The subscripts distinguish between the two rewards.

Solving the preceding equation for $D_{1}$ yields

$$
D_{1}=\left(A_{1} / A_{2}\right) *\left(k_{2} / k_{1}\right) * D_{2}+\left[\left(A_{1} / A_{2}\right)-1\right] / k_{1} \text {. }
$$

Thus, $D_{1}$ is a linear function of $D_{2}$ with a slope of $\left(A_{1} / A_{2}\right)$ * $\left(k_{2} / k_{1}\right)$ and an intercept of $\left[\left(A_{1} / A_{2}\right)-1\right] / k_{1}$. When $k_{2}=k_{1}$, as assumed in the analyses by Ainslie $(1975,1992)$, Mazur (1987), and Rodriguez and Logue (1988), the slope is greater than 1.0 if $A_{1}>A_{2}$. However, recent findings indicate that $k$ is a decreasing function of amount of reward. Given $A_{1}>A_{2}$ and an inverse relation between $k$ and amount, the slope will exceed 1.0 by even more than it will if $k$ is independent of amount. Note that if the ratio of the amounts is held constant and the absolute amounts are increased, the numerator of the intercept term remains constant and the denominator decreases. Thus, for equal amount ratios, larger intercepts are predicted for larger amounts if $k$ is a decreasing function of amount of reward. In contrast, if $k$ is assumed to be independent of amount, the intercept will be the same, regardless of the amounts, as long as the ratio is constant.

Alternatively, assume that the subjective value of a reward decreases exponentially with delay (Equation 2). If two rewards are equal in subjective value (i.e., $V_{1}=V_{2}$ ), then

$$
A_{1} * \exp \left(-k_{1} * D_{1}\right)=A_{2} * \exp \left(-k_{2} * D_{2}\right) \text {. }
$$

Taking the logarithms of both sides and solving the immediately preceding equation for $D_{1}$ yields

$$
D_{1}=\left(k_{2} / k_{1}\right) * D_{2}+\left[\ln \left(A_{1}\right)-\ln \left(A_{2}\right)\right] / k_{1} .
$$

Thus, $D_{1}$ is a linear function of $D_{2}$ with a slope of $\left(k_{2} / k_{1}\right)$ and an intercept of $\left[\ln \left(A_{1}\right)-\ln \left(A_{2}\right)\right] / k_{1}$. If $k_{2}=k_{1}$, as assumed in 
discounted utility theory as well as in the analyses of Ainslie, Mazur, and Rodriguez and Logue, the slope is 1.0. However, recent findings indicate that $k$ is a decreasing function of amount. Therefore, if $A_{1}$ is the larger amount, then $k_{2}>k_{1}$, and the predicted slope is greater than 1.0. Note that when the ratio of the amounts is held constant, the numerator of the intercept term is also constant, but the denominator, $k_{1}$, is a decreasing function of amount so that for equal amount ratios, larger amounts will lead to larger intercepts. In contrast, if $k$ is assumed to be independent of amount, the intercept will be the same, regardless of the amounts, as long as the ratio is constant.

These derivations show that, given recent demonstrations that larger amounts are discounted less steeply than smaller amounts, both hyperbolic discounting (Equation 1) and exponential discounting (Equation 2) lead to qualitatively similar predictions. More specifically, with regard to the delays that lead to subjective equality between two rewards, both hyperbolic and exponential discounting predict that the delay to the larger reward will be a linear function of the delay to the smaller with a slope greater than one and an intercept that, for equal amount ratios, is larger for larger amounts.

(Manuscript received February 2, 1994; revision accepted for publication May 11, 1994.) 